\title{
Sensory nerve conduction in the human spinal cord: epidural recordings made during scoliosis surgery
}

\author{
SJ JONES, MA EDGAR, AO RANSFORD \\ From the Medical Research Council and Royal National Orthopaedic Hospital, London
}

SUMMARY This report describes the waveform and properties of somatosensory evoked potentials recorded from various levels of the human spinal cord, with electrodes inserted into the epidural space and the stimulus delivered to the posterior tibial nerve at the knee. The object was to provide a means of monitoring spinal cord function during surgery for the correction of spinal deformities. The responses could be resolved into at least three components with different activation thresholds and different conduction velocities within the spinal cord $(45-80 \mathrm{~m} / \mathrm{s}$ approximately). The findings are in accord with recent studies, suggesting that the fast activity may be conducted in the dorsal spinocerebellar tract and the slower waves in the posterior columns.

Somatosensory evoked potentials (SEPs) were first recorded from the human spinal cord by Magladery et al, ${ }^{1}$ using needle electrodes inserted into the lumbar theca. Ertekin ${ }^{2}$ employed a similar technique at lumbar and low cervical level, and was able to record a conducted "tractus" potential at the latter site in response to stimulation of the posterior tibial nerve. Others (for example, Shimoji and coworkers $^{3}{ }^{4}$ ) have recorded mainly segmental responses with a flexible lead inserted into the epidural space. Segmental and conducted responses to lower limb stimulation have also been recorded from the surface of the skin, ${ }^{5-7}$ but the conducted "travelling" wave $^{5} 7$ is insufficiently reliable to be used as a clinical or research tool, at least in adult subjects.

Following the surgical treatment of spinal deformities by application of traction using a Harrington rod there is a small but significant incidence of neurological complications, including paraplegia. This has led a number of centres to develop techniques for monitoring the condition of the spinal cord during operation, by recording cortical ${ }^{8}$ or spinal $^{910}$ SEPs. In pilot studies we have found the potentials recorded from within the epidural space to be more stable and reliable than either spinal cord potentials recorded from the vertebral bone ${ }^{11}$ or cortical evoked potentials recorded from the scalp, ${ }^{8}$ and this paper presents the essentially normal

Address for reprint requests: Dr SJ Jones, National Hospital for Nervous Diseases, Queen Square, London WCIN 3BG, UK.

Received 8 November 1981

Accepted 12 December 1981 findings obtained prior to spinal distraction from patients without evidence of neurological lesions. The development of the technique and the changes that sometimes occur following the application of traction are to be described elsewhere (Edgar et al, in preparation).

\section{Methods}

All recordings were made in the operating theatres of the Royal National Orthopaedic Hospital, Stanmore, during treatment of scoliosis by posterior fusion and application of traction using a Harrington rod. The results described below were obtained from 67 cases (13 male, 54 female, mean age 15 years, range 11-27), all presenting with adolescent idiopathic scoliosis and all without evidence of neurological involvement. The patients received standard surgical anaesthesia and were ventilated following administration of curare and halothane. Blood pressure was maintained at less than $80 \mathrm{mmHg}$ using Trimetaphan.

The stimulus was a square wave impulse of duration $0.2 \mathrm{~ms}$ applied to the posterior tibial nerve at either knee by means of skin-surface electrodes. The intensity was adjusted so as to produce a direct motor response (plantar flexion of the foot) and was generally less than $100 \mathrm{~V}$. The stimulus frequency was usually 10 or 20 impulses/s. An earthing electrode (ECG plate) was secured to one leg on the proximal side of the stimulus. The active recording electrode was a blunted length of stainless steel needle, $3 \mathrm{~mm}$ long and $0.9 \mathrm{~mm}$ in diameter, attached to the end of a flexible lead insulated with PTFE to the same external diameter. After exposure of the upper thoracic vertebrae this was introduced into the dorsal epidural space by means of a 16-gauge catheter (Abbocath-T) and advanced cephalad by $1-3 \mathrm{~cm}$. The reference electrode was a stainless steel needle inserted into the muscle alongside the epidural 446 
recording site. Recordings were also obtained from lower thoracic and lumbar levels in 11 cases, and in four cases the stimulus was additionally delivered to the posterior tibial nerve at the ankle.

The evoked potentials were routinely amplified over a fairly narrow bandwidth $(200 \mathrm{~Hz}-2 \mathrm{kHz})$, averaged and recorded on photographic or heat-sensitive paper. The majority of early recordings were obtained using a Medelec MS6 EMG machine in which the averager module was an analogue device containing 100 memory addresses. These were distributed over 10 or $20 \mathrm{~ms}$ of the input sweep, starting 8-14 ms after the stimulus. Later a few recordings were obtained using a more modern MS6 with 1024 memory addresses and a digital data store. Most recent recordings were obtained with a Medelec MS91, containing 1000 memory addresses, a data store and a "normalised" display in which the amplification factor was independent of the number of sweeps accumulated.

\section{Results}

Recordings were obtained at T3 level or above in response to stimulation of 125 limbs in 65 patients (two patients were monitored at mid-thoracic level). No evoked activity was seen during the first 10 or $15 \mathrm{~ms}$, but after this there occurred a sequence of positive and negative deflections with a duration of approximately $6 \mathrm{~ms}$ (fig 1 ). The waveform could usually be resolved into at least three components, the first being a triphasic (positive/negative/positive) wave resembling a conventional nerve action potential, the second a fairly sharp negativity and the third a broader negative wave. The mean latencies of the three negative peaks across all subjects were $15 \cdot 2$, 17.0 and $19.2 \mathrm{~ms}$ (see table 1), correlated with the patients' age and body height. The amplitude of the response, measured from the deepest trough to the highest peak (usually the second positive deflection to the second negativity) was between 1 and $6 \mu \mathrm{V}$, with a mean of 2.6 $\mu \mathrm{V}$. Provided the position of the electrode was undisturbed the waveform generally remained fairly constant within the individual, although a slight latency increase was noticed throughout the operation. The potentials recorded in response to stimulation of the left and right legs usually differed by less than $0.5 \mathrm{~ms}$ in latency and $30 \%$ in amplitude, but differences as great as $1 \mathrm{~ms}$ and $60 \%$ were occasionally observed (fig 1 ).

Table 1 Somatosensory evoked potentials recorded from the epidural space at or above T3 level following posterior tibial nerve stimulation at the knee (65 cases).

\begin{tabular}{llll}
\hline Amplitude $(\mu v)$ & \multicolumn{4}{l}{ Negative peak latencies $(m s)$} \\
\cline { 2 - 4 } & 1 & 2 & 3 \\
\hline $2.6 \pm 1 \cdot 1$ & $15.2 \pm 1 \cdot 3$ & $17.0 \pm 1.4$ & $19 \cdot 2 \pm 1 \cdot 7$ \\
(range 1.0-6.0) & $(12.5-17.5)$ & $(13.5-19 \cdot 0)$ & $(15-22)$ \\
\hline
\end{tabular}

On account of the fairly high level of background noise the potentials were usually amplified over a narrow bandwidth from $200 \mathrm{~Hz}$ to $2 \mathrm{kHz}$. With a wider bandwidth of $20 \mathrm{~Hz}$ to $10 \mathrm{kHz}$ there was a slight increase in amplitude (most marked for the broader third component) and improvement in the resolution of very brief subcomponents (see fig 1 ).

$\mathrm{CH}$ age 17 recording at $\mathrm{T} 2$

$200 \mathrm{~Hz}-2 \mathrm{kHz}$
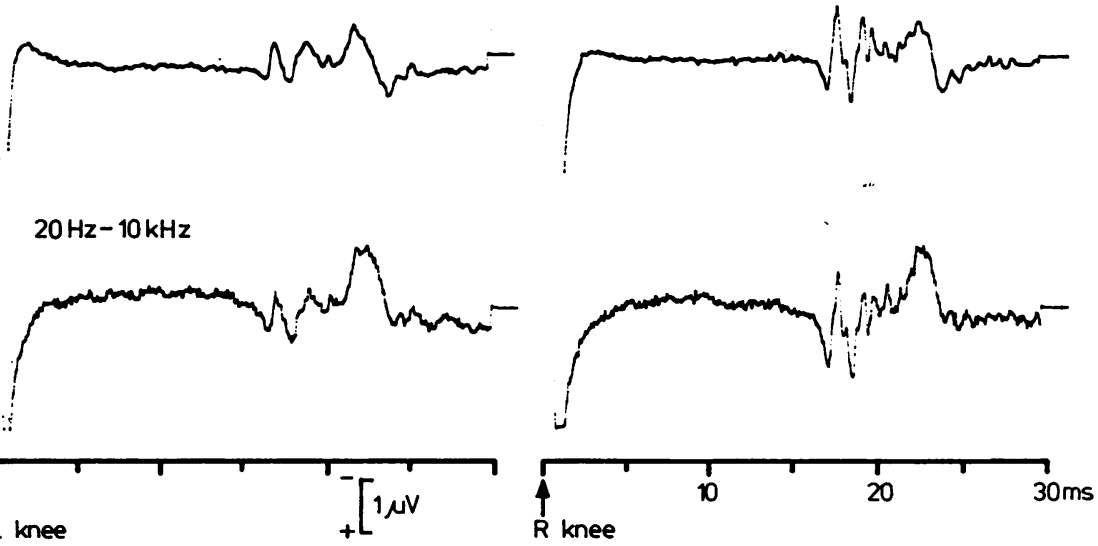

Stimulus :

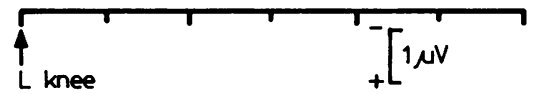

Fig 1 Epidural SEPs recorded at T2 level following stimulation of the left and right posterior tibial nerve at the knee. The earlier components are approximately $50 \%$ smaller on the left. The main effect of a wider recording bandwidth is to increase the amplitude of the third component. 
In order to facilitate rapid detection of any waveform changes which might occur, the chosen rate of stimulation was as fast as permitted by technical and physiological factors. There was found to be very little difference between the responses recorded at 2, 5, 10 and 20 impulses/s (fig 2), and at 50 impulses/s only a slight amplitude reduction was observed, mainly of the second component in this subject. The waveform appeared to be little affected by fluctuations in the level of anaesthesia and blood pressure. When the stimulus voltage was gradually increased from 0 to $100 \mathrm{~V}$ the three main components appeared and attained maximal amplitude at different intensities, the thresholds ranging from about $25 \mathrm{~V}$ for the first to $80 \mathrm{~V}$ for the last component (fig 3).

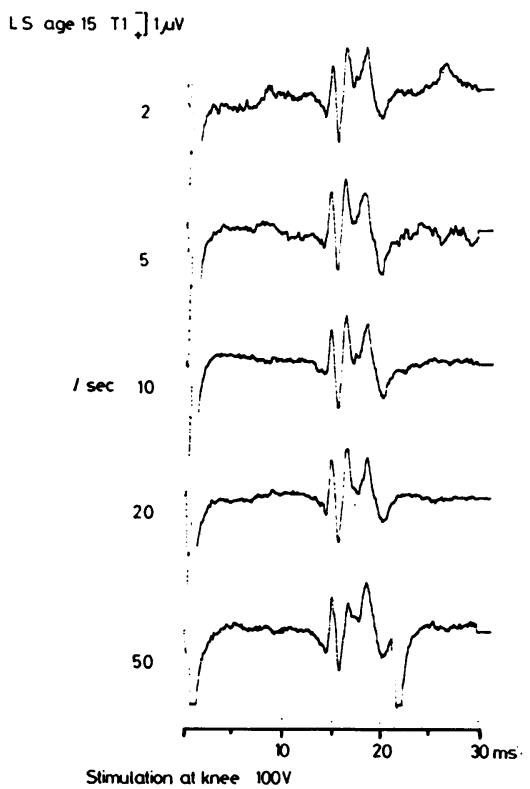

Fig 2 Epidural SEPs recorded at TI level following stimulation at the knee; 2, 5, 10, 20 and 50 impulses/s. At the fastest rate the responses are fractionally increased in latency, and the second negative peak is of smaller amplitude.

Although it was not possible to identify with any degree of certainty equivalent components in the response to stimulation at the knee and in the much smaller and less well-defined waveform evoked by stimulation at the ankle, the latency difference between the two waveforms suggested a peripheral nerve conduction velocity between 35 and $50 \mathrm{~m} / \mathrm{s}$ for the fibres concerned (fig 4). The apparent overall conduction velocity of the fastest component from the knee to T2 vertebral level was between 50 and
DF age 11

30

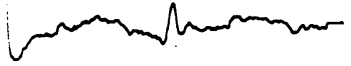

$40 \mathrm{~V}$

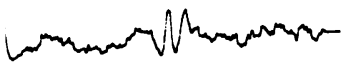

$60 \mathrm{~V}$

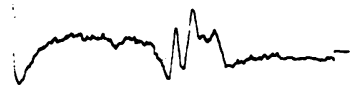

$100 \mathrm{v}$
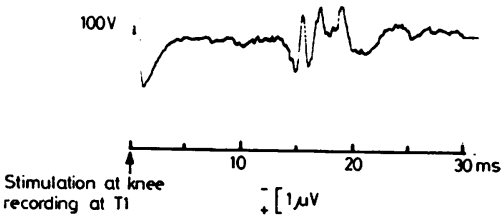

Fig 3 Epidural SEPs recorded at TI level following stimulation at the knee; 30, 40, 60 and $100 \mathrm{~V}$. The three major components have clearly different excitation thresholds, the later waves requiring more intense stimuli.

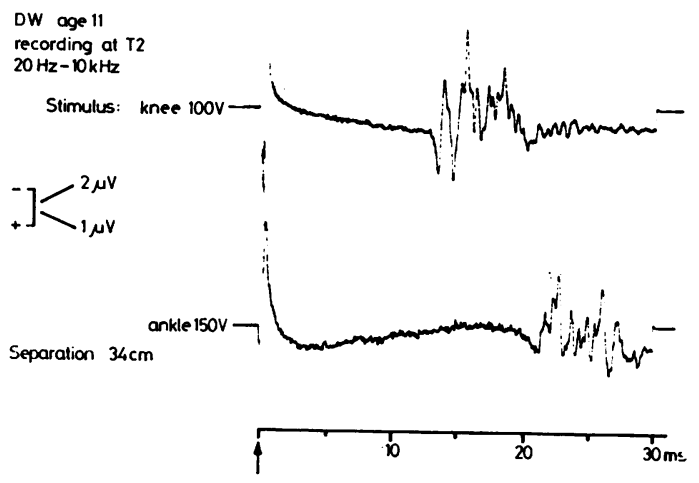

Fig 4 Epidural SEPs recorded at T2 level following stimulation at the knee and the ankle. Equivalent components cannot be identified with certainty in the two recordings, but the overall difference in latency suggests a peripheral conduction velocity of $35-50 \mathrm{~m} / \mathrm{s}$. Note the multiplicity of brief components resolved by a wide recording bandwidth.

$60 \mathrm{~m} / \mathrm{s}$, and that of the slowest around $45 \mathrm{~m} / \mathrm{s}$. Corresponding values were slightly slower (by up to $5 \mathrm{~m} / \mathrm{s}$ ) when measured from the ankle to T2. Assuming that the break-up of the SEP into distinct components was at least partially due to separate fibre groups in the peripheral nerve (the evidence being the 
different activation thresholds), this suggests either that conduction in some fibres becomes faster over peripheral nerve segments proximal to the knee and within the spinal cord, or that the fast conducting fibres responsible for the shortest latency components are not present in any quantity at the level of the ankle.

In 11 cases it was possible to record at two or more levels of the spine, following stimulation at the knee or the ankle. At low thoracic and lumbar levels the responses were of larger amplitude and shorter duration (figs 5 and 6). Often it was not possible to identify equivalent components at both recording sites, particularly if they were widely spaced, but comparing the latencies of the initial positive deflection, the point of transition from positive to negative polarity and the first negative peak consistently gave conduction velocity values between 60 and $80 \mathrm{~m} / \mathrm{s}$ following stimulation at the knee. In three cases the waveform recorded at mid-thoracic level was similar to that obtained rostrally, and it was possible to identify three negative peaks following stimulation at the knee and (more doubtfully) the ankle. For the subject illustrated in fig 6 the knee responses were apparently conducted at around 75,65 and $50 \mathrm{~m} / \mathrm{s}$, but from the ankle the entire waveform had an apparent conduction velocity between 45 and $55 \mathrm{~m} / \mathrm{s}$ within the thoracic spinal cord.

\section{Discussion}

In a great many centres the procedure whereby an

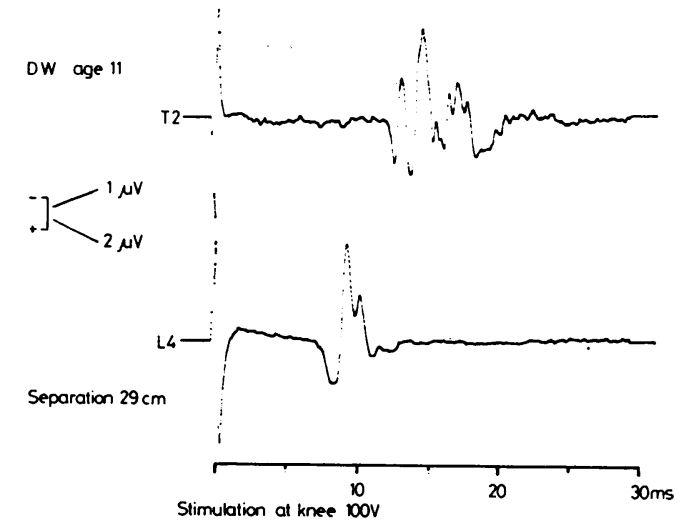

Fig 5 Epidural SEPs recorded at T2 and L4 level following stimulation at the knee. Equivalent components are not identifiable, but the apparent conduction velocity of the initial positivity and negativity is between 65 and $75 \mathrm{~m} / \mathrm{s}$.

electrode is introduced into the epidural space for recording evoked potentials from the spinal cord is considered too invasive to be acceptable for use with conscious subjects or patients. However, during spinal surgery there is a need for a sensitive method of monitoring the integrity of the spinal cord, and under sterile conditions with the vertebrae already exposed the placement of an epidural electrode is a relatively insignificant manoeuvre. It is not intended, in this discussion, to consider the merits and weaknesses of the various spinal and cortical SEP

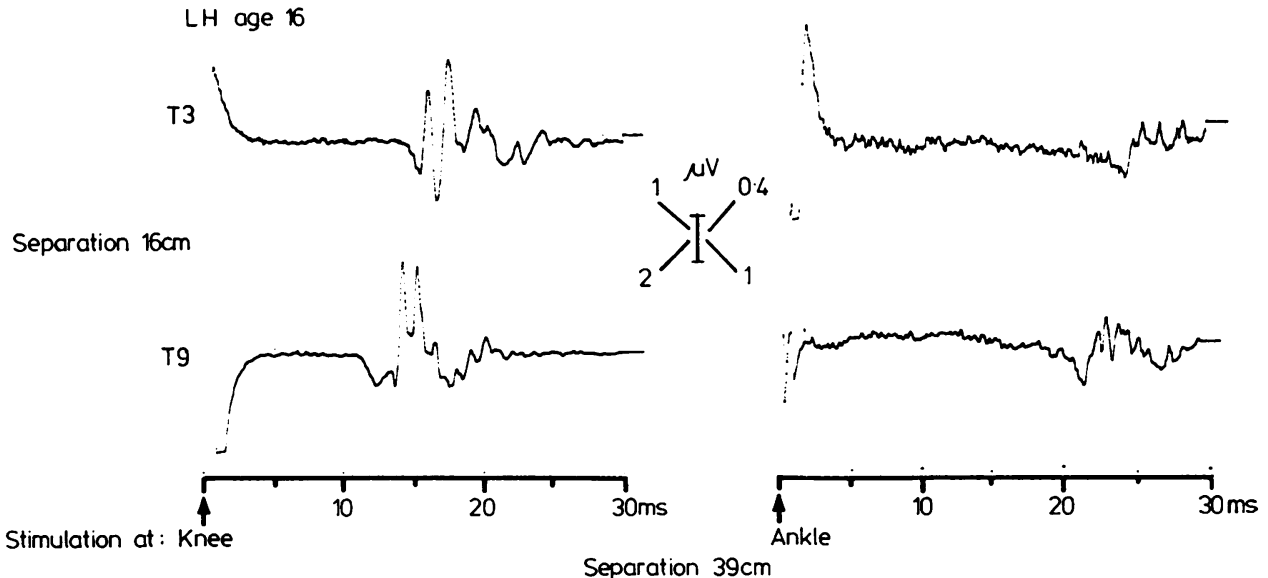

Fig 6 Epidural SEPs recorded at T3 and T9 following stimulation at the knee and the ankle. Assuming the equivalence of the three main peaks present at the two recording sites the spinal cord conduction velocities were approximately 75,65 and $50 \mathrm{~m} / \mathrm{s}$ following stimulation at the knee, and $45-55 \mathrm{~m} / \mathrm{s}$ following stimulation at the ankle. This suggests that the components of the ankle response cannot be due to the same population of nerve fibres as the first two peaks of the knee response, but may be equivalent to the third component from the knee. 
techniques which have been developed for the purpose of spinal cord monitoring, but our pilot studies led us to prefer the epidural approach, by which a complex but stable SEP can be recorded without (in our experience) giving rise to medical complications.

In the earliest published account of evoked activity in the human spinal cord Magladery et al ${ }^{1}$ described mainly segmental (root entry zone) responses recorded in the lumbar region, and propagated potentials generated in the anterior and posterior roots of the cauda equina. The amplitude of the response fell off rapidly at more rostral levels, but there was also a slight increase in latency, suggesting a cord conduction velocity in the order of $30 \mathrm{~m} / \mathrm{s}$. This was slightly slower than that of the peripheral sensory fibres concerned, which conducted at $45-50 \mathrm{~m} / \mathrm{s}$ between the ankle and the knee.

Using needle electrodes placed intrathecally at low cervical as well as lumbar level, Ertekin ${ }^{2}$ studied the properties of a "tractus" potential which had a mean negative peak latency of $26 \mathrm{~ms}$ at the cervical electrode, a mean duration of $16 \mathrm{~ms}$ and appeared to be propagated at $30-50 \mathrm{~m} / \mathrm{s}$ between the two recording sites. The potential was usually triphasic in form, but was occasionally preceded by a small negativity.

Shimoji et $a l^{34}$ recorded mainly segmental responses from within the epidural space, and reported that the potentials which could be detected a few segments rostral to the appropriate root entry zone were of very much smaller amplitude. However, two propagated waves were described ${ }^{3}$ which, in marked contrast to the "tractus" potential, 2 were apparently conducted at $120-130 \mathrm{~m} / \mathrm{s}$ from T10 to T3. In a later study ${ }^{4}$ a waveform consisting of a fast positive/negative spike followed by a broader negativity was recorded with a latency of $5-8 \mathrm{~ms}$ in the cervical region, following direct electrical stimulation of the cauda equina. A somewhat similar wave with a reported conduction velocity of about $85 \mathrm{~m} / \mathrm{s}$ was described by Shimizu et al ${ }^{12}$.

Tsuyama et $a l^{9}$ adopted a technique of direct spinal cord stimulation, with both stimulating and recording electrodes located in the dorsal epidural space. Both ascending and descending potentials were recorded, each consisting of a bi-or triphasic spike followed by a broader negativity, conducted at around 73 and $47 \mathrm{~m} / \mathrm{s}$ respectively. When the stimulating electrode was located near the midline the late negativity had a lower threshold of excitation, whereas a more lateral placement caused the triphasic wave to be evoked at lower stimulus intensities. It was concluded that the two components were likely to be conducted through different parts of the cord, the triphasic wave in the lateral tracts and the later negativity in the posterior columns.
Support for this hypothesis was provided by partial spinal cord transection in decerebrate cats, with the result that the triphasic wave was found to be abolished by a lesion of the lateral white matter while the late negativity was attenuated by section of the posterior columns.

With an electrode located in the subarachnoid space, Tamaki et al ${ }^{10}$ recorded a descending potential at the level of the conus medullaris following epidural stimulation of the upper thoracic spinal cord. In its configuration and properties this wave was very similar to that described by Tsuyama et al. ${ }^{9}$ It was stable to administration of anaesthetics, but was sensitive to accidental cord compression during surgery and to application of excessive traction. The severity of any postoperative neurological symptoms was generally found to reflect the degree to which the response failed to recover following reversal of the surgical manoeuvre.

Although much of this data is apparently conflicting (table 2), a few of the recent studies are in some measure of agreement. The consensus appears to be that the fastest evoked spinal cord activity, recorded as a triphasic wave and perhaps originating in the lateral tracts, is conducted at $65-80 \mathrm{~m} / \mathrm{s}$, while slower potentials possibly arising in the posterior columns have conduction velocities ranging from about 30 to $50 \mathrm{~m} / \mathrm{s}$.

Table 2 Published values of sensory conduction velocity in the human spinal cord (lumbar or low thoracic to high thoracic)

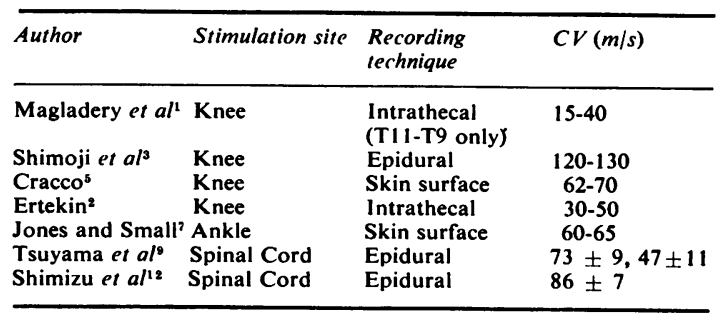

On the surface of the skin only a single "travelling wave" component has been reported ${ }^{5} 7$ with a conduction velocity of $60-70 \mathrm{~m} / \mathrm{s}$. This may represent the envelope of the faster and slower potentials recorded epidurally, but it is surprising that the conduction velocities were similar whether the stimulus was delivered to the knee ${ }^{5}$ or to the ankle. ${ }^{?}$

In our experience the fastest spinal cord potentials recorded epidurally were evoked by low intensity stimulation of the posterior tibial nerve at the knee, but not by any intensity of stimulation at the ankle. This suggests that the peripheral afferent fibres concerned are likely to be those of Groups $1 \mathrm{a}$ and $1 \mathrm{~b}$, which derive mainly from muscle spindles and 
tendon organs and hence are likely to be much more numerous at the level of the knee than at the ankle. Some of these fibres may pass directly into the posterior columns, but the majority are likely to synapse in the dorsal horns, on cells which give rise to the dorsal spinocerebellar tract. This contains large diameter axons which respond to discrete muscle contraction as well as to stimulation of touch and pressure receptors. ${ }^{13}$

The potentials recorded in the upper thoracic region following peripheral nerve stimulation were of very low amplitude compared with epidurallyrecorded responses to direct electrical stimulation of the spinal cord. ${ }^{910}$ They were, however, stable in terms of amplitude, latency and waveform, and easy enough to record (with the aid of modern signal averaging devices) to permit their use as a research tool and as a routine means of monitoring spinal cord integrity during potentially injurious surgery. Some advantages of peripheral nerve over spinal cord stimulation techniques may be innumerated as follows: (1) the safety factor of having only one electrode in close proximity to the spinal cord; (2) convenience for the surgeon, with only one electrode pair to manipulate; (3) a more "natural" afferent volley, and (4) the likelihood of fewer problems with directly conducted stimulus artifacts. Against this must be set the lower recorded amplitudes and the possibility (seldom encountered in our experience) of deteriorating contact between the stimulating electrode and the peripheral nerve.

The authors thank Ms Linda Carter for technical assistance, and Action Research for the Crippled Child for provision of an equipment grant.

\section{References}

${ }^{1}$ Magladery JW, Porter WE, Park AM, Teasdall RD. Electrophysiological studies of nerve and reflex activity in normal man; (iv) The two-neurone reflex and identification of certain action potentials from spinal roots and cord. Bull Johns Hopkins Hosp 1951; 88:499-519.

${ }^{2}$ Ertekin C. Studies on the human evoked electrospinogram; (ii) The conduction velocity along the dorsal funiculus. Acta Neurol Scand 1976;53:21-38.

${ }^{3}$ Shimoji K, Kano T, Higashi H, Morioka T, Henschel EO. Evoked spinal electrograms recorded from epidural space in man. $J$ Appl Physiol 1972;33: 468-71.

4 Shimoji K, Shimizu H, Maruyama Y. Origin of somatosensory evoked responses recorded from the cervical skin surface. J Neurosurg 1978;48:980-4.

${ }^{5}$ Cracco RQ. Spinal evoked response: peripheral nerve stimulation in man. Electroencephalogr Clinical Neurophysiol 1973;35:379-86.

- Delbeke J, McComas AJ, Kopec SJ. Analysis of evoked lumbosacral potentials in man. $J$ Neurol Neurosurg Psychiatry 1978;41:293-302.

7 Jones SJ, Small DG. Spinal and subcortical evoked potentials following stimulation of the posterior tibial nerve in man. Electroencephalogr Clinical Neurophysiol 1978;44:299-306.

${ }^{8}$ Nash CL, Lorig RA, Schatzinger LA, Brown RH. Spinal cord monitoring during operative treatment of the spine. Clin Orthop 1977;126:100-5.

${ }^{9}$ Tsuyama N, Tsuzuki N, Kurokawa T, Imai T. Clinical application of spinal cord action potential measurement. Int Orthop 1978;2:39-46.

10 Tamaki T, Tsuji H, Inoue S-I, Kobayashi H. The prevention of iatrogenic spinal cord injury utilizing the evoked spinal cord potential. Int Orthop 1981;4: 313-7.

11 Nordwall A; Axelgaard J, Harada Y, Valencia P, McNeal DR, Brown JC. Spinal cord monitoring using evoked potentials recorded from feline bone. Spine 1979;4:486-94.

12 Shimizu H, Shimoji K, Maruyama Y, Sato H, Harayama $H$, Tsubaki $T$. Slow cord dorsum potentials elicited by descending volleys in man. $J$ Neurol Neurosurg Psychiatry 1979;42:242-6.

${ }^{13}$ Lundberg A, Oscarsson O. Functional organisation of the dorsal spino-cerebellar tract in the cat; (vii) Identification of units by antidromic activation from the cerebellar cortex with recognition of five functional subdivisions. Acta Physiol Scand 1960;50: 356-74. 